\title{
Energetic ions generated by laser pulses: A detailed study on target properties
}

\author{
M. Roth, A. Blazevic, M. Geissel, and T. Schlegel \\ Gesellschaft für Schwerionenforschung mbH, Planckstrasse 1, Darmstadt 64291, Germany \\ T.E. Cowan and M. Allen \\ General Atomics, P.O. Box 85608, San Diego, California 92186-5608 \\ J.-C. Gauthier, P. Audebert, and J. Fuchs \\ Laboratoire pour l'Utilisation des Lasers Intenses, Palaiseau 91128, France
}

J. Meyer-ter-Vehn, M. Hegelich, S. Karsch, and A. Pukhov

Max-Planck-Institut für Quantenoptik, Garching, Germany

(Received 15 November 2001; published 4 June 2002)

\begin{abstract}
We present the results of a detailed study on the acceleration of intense ion beams by relativistic laser plasmas. The experiments were performed at the $100 \mathrm{TW}$ laser at the Laboratoire pour L'Utilisation des Lasers Intenses. We investigated the dependence of the ion beams on the target conditions based on theoretical predictions by the target normal sheath acceleration mechanism. A strong dependence of the ion beam parameters on the conditions on the target rear surface was found. We succeeded in shaping the ion beam by the appropriate tailoring of the target geometry and we performed a characterization of the ion beam quality. The production of a heavy ion beam could be achieved by suppressing the amount of protons at the target surfaces. Finally, we demonstrated the use of short pulse laser driven ion beams for radiography of thick samples with high resolution.
\end{abstract}

DOI: 10.1103/PhysRevSTAB.5.061301

PACS numbers: 41.75.Jv, 52.59.-f, 52.38.-r, 52.70.-m

\section{INTRODUCTION}

Recently, the production of intense ion beams of laser irradiated targets has been observed [1-4]. Some experiments, using ultraintense short pulse lasers [5] with intensities exceeding $10^{19} \mathrm{~W} / \mathrm{cm}^{2}$, have shown collimated beams of protons that have a very low emittance, while reaching energies of up to $50 \mathrm{MeV}$ [6], which is understood as rear surface emission accelerated by the target normal sheath acceleration (TNSA) mechanism [7]. This ion beam generation is attributed to electrostatic fields produced by hot electrons acting on protons from adsorbed water vapor and hydrocarbons $[8,9]$. Relativistic electrons generated from the laser-plasma interaction, having an average temperature of several $\mathrm{MeV}$, envelope the target foil and form an electron plasma sheath on the rear, nonirradiated surface. The electric field in the sheath can reach $>10^{12} \mathrm{~V} / \mathrm{m}$, which field ionizes atoms on the surface and accelerates the ions very rapidly normal to the rear surface. Protons, having the largest charge-to-mass ratio, are preferentially accelerated in favor of heavier ions over a distance of a few microns, and up to tens of MeV. This forms in a collimated beam with an approximately exponential energy distribution with 5-6 MeV. This acceleration mechanism makes these intense ion beams highly interesting for many applications, especially if one can collimate or focus the beam by shaping the target, as suggested by numerical calculations $[10,11]$. Because of the dependence of the ion beam on the formation of the sheath, this process should reveal information about the electron transport through the target. The transport of relativistic electrons through the target is an extensively studied area of research [12-15] because of its relevance not only for ion acceleration, but especially for the concept of fast ignition [16-18] in inertial confinement fusion. The transport of large currents through the target, the onset of beam filamentation, and the dependence on the target conductivity (with respect to the onset of return currents) are essential for a detailed understanding of the ion accelerating mechanism. We expect that details of the ion acceleration will also depend on the target material and surface conditions. Therefore we carried out experiments to investigate in detail the influence of these target parameters on the ion beam production.

\section{EXPERIMENTS}

The experiments presented in this paper were performed with the $100 \mathrm{TW}$ laser at Laboratoire pour l'Utilisation des Lasers Intenses (LULI). Pulses of up to $30 \mathrm{~J}$ at $300 \mathrm{fs}$ pulse duration at $\lambda=1.05 \mu \mathrm{m}$ were focused with an $f / 3$ off-axis parabolic mirror onto free standing target foils at normal incidence, at intensities up to $5 \times 10^{19} \mathrm{~W} / \mathrm{cm}^{2}$. The focal spot diameter (FWHM) measured in vacuum was about $8 \mu \mathrm{m}$. Amplified spontaneous emission (ASE) occurred 2 ns before the main pulse at a level of $10^{-7}$ of the main pulse energy and preformed a plasma.

The diagnostic setup is depicted in Fig. 1. The freestanding target was probed by a frequency doubled laser beam parallel to the surface to determine the plasma conditions on the front and rear surfaces. A stack of 
radiochromic film (RCF) was positioned a few $\mathrm{cm}$ behind the target to measure the spatial beam profile. Because of the pronounced energy loss of ions at the end of their range (Bragg peak), different layers of the RCF pack allow the imaging of the ion beam at different energies. The RCF changes through polymerization of a diacetylene active layer, from transparent to dark blue, in proportion to the absorbed dose (rads) of ionizing radiation $(1 \mathrm{rad}=100 \mathrm{erg} / \mathrm{g})$. We modeled the response of the RCF package with the SRIM [19] Monte Carlo ion transport code, assuming the beam to be protons, and obtained response functions for the layers of RCF similar to the ones presented in Ref. [2]. A slot in the center of the RCF allowed a free line of sight for the charged particle spectrometers fielded at $0^{\circ}, 6^{\circ}$, and $13^{\circ}$ to provide the energy distribution of the emitted electrons and ions.

Two absolutely calibrated, permanent magnetic ion spectrometers were mounted at a distance of about $1 \mathrm{~m}$ from the target covering a solid angle of $5 \times 10^{-6} \mathrm{sr}$. The electrons and protons were recorded in nuclear emulsion track detectors which allow single particle detection without being overwhelmed by the blinding $\mathrm{x}$-ray flash from the laser plasma. The use of an additional x-ray film layer extended the dynamic range to higher particle fluxes. A protective light tight paper in front of the emulsion and x-ray film stopped protons below $1.8 \mathrm{MeV}$. We extended the spectral range to lower proton energies using CR-39 in the low energy range of the dispersion plane. By etching the CR-39 detectors in sodium hydroxide, the material damage caused by the impacts of ions above a threshold of a few hundred $\mathrm{keV}$ become visible. Microscopic scanning provides position, as well as the size of the impact, which is proportional to the atomic number $Z$ of the ion. Thus, protons produce much smaller pit sizes than heavy ions.

As a complementary measure of the total yield of protons we used a titanium catcher foil, which was placed in the path of the proton beam. The ${ }^{48} \mathrm{Ti}$ is transmuted by a $(p, n)$ reaction by protons above a sharp reaction threshold at $\sim 5 \mathrm{MeV}$ to an excited state of the ${ }^{48} \mathrm{~V}$ isotope. Using a low-background Ge detector we observed the gamma deexcitation lines of the ${ }^{48} \mathrm{Ti}(p, n)^{48} \mathrm{~V}$ reaction, which pro-

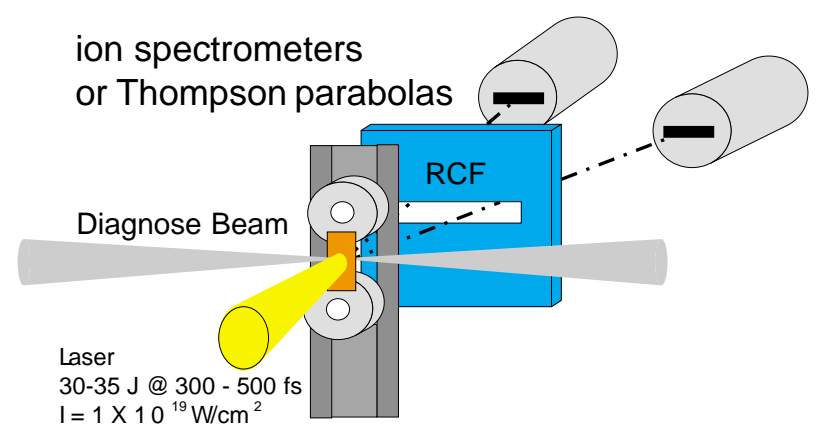

FIG. 1. (Color) Experimental setup. The freestanding target is irradiated at normal incidence. A slit in the radiochromic film (RCF) gives a line of sight for the particle spectrometer. vided the total activation and therefore the yield of protons above the reaction threshold of $\sim 5 \mathrm{MeV}$.

To detect heavy ions accelerated from the rear surface of the relativistic-laser illuminated targets, we substituted two high resolution Thompson parabolas in replacement of one of the charged-particle spectrometers. The parallel electric and magnetic fields in the Thompson parabolas discriminated ions with respect to their momentum and charge-to-mass ratio, at the plane of the CR-39 track detectors. Careful analysis of the scanned CR-39 detectors then provides absolute numbers of the ions with respect to their kinetic energy and charge state. In addition to the ion and laser beam detectors, a silver activation neutron detector was fielded close to the target chamber determining the neutron yield for the different experiments. The silver is activated by neutron impact capture and $\beta^{-}$decay with a half-life of $28 \mathrm{sec}$, which is detected in a scintillator and recorded by a photomultiplier tube (PMT).

\section{RESULTS}

\section{A. Hydrodynamic target stability}

For the effective acceleration of the ions, an undisturbed back surface of the target is crucial to provide a sharp ion density gradient as the accelerating field strength is proportional to $T_{\text {hot }} / e l_{0}$, where $T_{\text {hot }}$ is the temperature of the hot electrons and $l_{0}$ is the larger of either the hot-electron Debye length or the ion scale length of the plasma on the rear surface. The preceding ASE launches a shock wave into the target which causes a destruction of the acceleration sheath. Therefore the target thickness was chosen to guarantee an undisturbed rear surface based on numerical calculations using the hydrocode MULTI [20]. The result of the simulation is shown on the left-hand side of Fig. 2. The inward propagating shock wave is clearly visible. It reached the rear surface at about $8 \mathrm{~ns}$ after the onset of the prepulse. Thus, the targets should maintain an undisturbed back surface for a $5 \mathrm{~ns}$ prepulse. When we applied a prepulse at a contrast ratio of $10^{-7}$ of the main pulse $10 \mathrm{~ns}$ before the main pulse, the maximum energy of the protons dropped to $2 \mathrm{MeV}$ from the typical $10-20 \mathrm{MeV}$ range typical of low-prepulse shots. This is in good agreement with the мULTI calculations, which indicate that in $10 \mathrm{~ns}$ a shock wave launched by the prepulse penetrates the target and causes a rarefaction wave that diminishes the density gradient on the back and therefore drastically reduces the accelerating field. The inward moving shock wave also alters the initial conditions of the target material due to shock wave heating and therefore changes, e.g., the target density and conductivity. Because of its relevance to the electron transport, we chose the target thickness such that a considerable fraction of the target was still in its unperturbed, initial condition. Figure 2 also shows interferometric measurements of the target surface with and without the additionally applied prepulse. The interferometry detects the plasma density conditions on the front and rear surfaces 


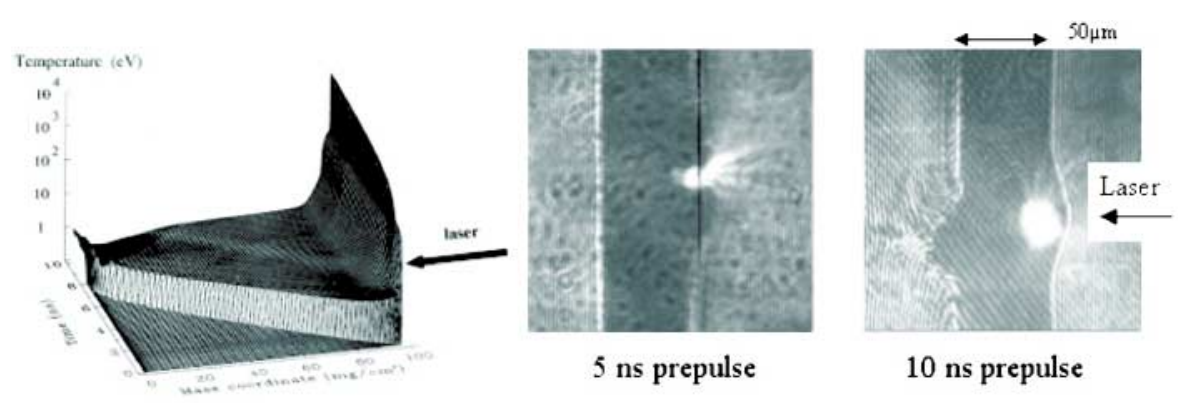

FIG. 2. Left: simulation of the shock wave launched by the prepulse. The shock wave reaches the rear surface at about 7 ns. Center: interferometric images of the plasma conditions on the front and rear target surfaces. Right: perturbation of the rear surface due to prepulse induced shock wave breakout. No protons were detected.

simultaneously. In the figure, the laser is incident from the right (note that the bright spot is $2 \omega$ emission from the laser plasma during the pulse). As shown in Fig. 2, the front surface always shows the blowoff plasma, extending up to about $200 \mu \mathrm{m}$, caused by the ASE. In the absence of a prepulse (left image) the rear surface is unperturbed (no plasma formation could be detected within the resolution limit of the interferometry of a few micrometers) and a strong high-energy proton signal could be detected on the RCF. In agreement with the TNSA predictions, when we observed the presence of an extended plasma (about $50 \mu \mathrm{m}$ ) at the rear surface due to the applied prepulse, no protons above the detection threshold of our RCF $(\sim 2 \mathrm{MeV})$ could be measured. This result is also in excellent agreement with recent experiments using a second laser to generate a plasma at the rear target surface [21].

\section{B. Angular dependence}

The angular dependence of the energy distribution of the proton beam was measured with two ion spectrometers positioned at an angle of $0^{\circ}$ and $13^{\circ}$, respectively. The measured spatial distributions of protons on the dispersion plane were deconvoluted (with respect to the entrance aperture shape) [22] and corrected for the spectrometer dispersion. The energy of the protons emitted normal to the target rear surface extended up to $25 \mathrm{MeV}$. The maximum energy of the protons dropped to about $13 \mathrm{MeV}$ at an angle of $13^{\circ}$, consistent with a $2 \mathrm{D}$ model of the sheath acceleration process. The spectral shape of each proton energy distribution is generally continuous up to the cutoff energy, in agreement with the electrostatic sheath acceleration mechanism, as well as previous observations in experiments with the LLNL PETAWATT laser [6]. The best fit to the spectrum obtained by the ion spectrometers, as well as to the spectral information extracted from the stacked RCF packages, was obtained by using a two component exponential distribution with 2 and $6 \mathrm{MeV}$, respectively. Details about the angular dependence of the ion beam and the origin of occasionally observed narrow features in the spectral distribution, caused by the segregation of different ion species, are beyond the scope of this paper and will be published elsewhere.

\section{Yield, surface dependence}

Previous experiments using gold targets of similar size, performed at the LLNL PETAWATT laser, revealed a smooth, collimated beam of protons perpendicular to the rear surface. The origin of the protons was found to be contaminant layers of water vapor and hydrocarbons. The total yield of protons could be increased significantly using plastic targets, due to acceleration of protons from the bulk material; however, the laminarity of the beam was largely disrupted, with the spatial pattern of the accelerated protons exhibiting a large degree of filamentarylike structure. We considered several possible reasons for this behavior, including quality of the plastic versus gold material, ion-acoustic instabilities during the ion expansion, and conductivity of the bulk material or of the target rear surface. To investigate the influence of such target conditions on the creation of a collimated ion beam, we varied the target composition and structure of the rear surface.

We used thin $(48 \mu \mathrm{m})$ targets of gold with either a flat or structured rear surface. The proton beam ejected from the rear surface is shown in the two left-hand pictures of Fig. 3. The results showed a clear dependence of the spatial uniformity of the proton beam on the structure of the back surface. In contrast to the homogenous, collimated beam from the gold target, protons emitted from the structured gold rear surface showed filaments. To discriminate between conductivity and surface quality effects, we next used $\sim 100 \mu \mathrm{m}$ plastic and glass targets. The results of the glass and plastic targets were even more pronounced. While the flat surfaces of glass and plastic yielded a strong, but filamented proton beam (Fig. 3, third image), there were no protons detected above $1 \mathrm{MeV}$ from the roughened targets (Fig. 3, right-hand image). The similar beam patterns obtained from plastic and glass targets exclude the origin (surface or bulk) of the protons to be the reason for the onset of the filamentary structures. In contrast, due to the strong coupling of the ion acceleration mechanism to 

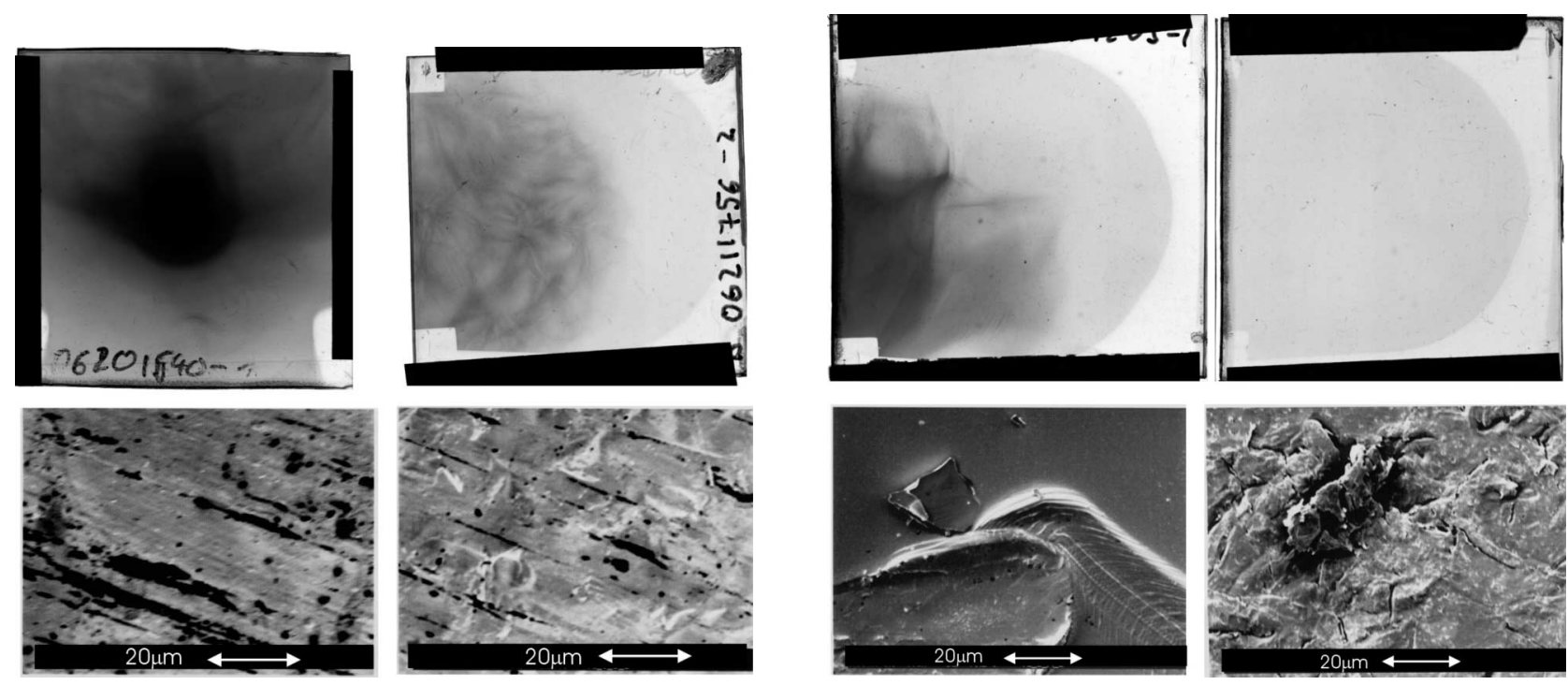

FIG. 3. Proton emission from smooth and roughened rear surfaces of gold (two left-hand columns) and plastic targets. The roughened surface in the case of gold leads to the onset of filamentation. No protons could be detected from the roughened plastic targets due to the destroyed surface. The scanning electron microscope (SEM) images show the corresponding structures of the surfaces [note that the structure visible for the smooth plastic target (third, lower inset) was an artifact to focus the SEM]. The overall surface of the target was similar to the upper right-hand part.

the electron distribution at the rear surface of the target, the smooth, laminar beam quality from metal, i.e., conductor targets, indicates a rather homogenous electron transport through the target. Insulating material seems to disrupt the electron transport, which causes filamentation of the electron distribution and therefore also a nonhomogenous ion acceleration. The dependence of the electron transport on target conductivity has been observed by other research groups as well [23]. As mentioned above, the preceding ASE leads to a shock wave that preheats the target, thereby changing the conductivity of the insulating targets. However, in our experiments the target thickness has been chosen such that a considerable fraction of the target material was still in the cold, solid state by the time of arrival of the main pulse.

Using a scanning electron microscope (SEM) we examined the structure of the target surfaces. The images are shown as the corresponding lower insets in Fig. 3. Structuring the gold surface maintained a smooth surface with hills and valleys, visible as bright shadows on the lower, second inset of Fig. 3. The surface of the plastic and glass targets was largely destroyed by numerous cracks. The different behavior of the structured gold, glass, and plastic targets can be understood within the context of the TNSA model. When the material on the rear surface is exposed to the strong electric field generated by the electron plasma sheath, it is field ionized instantaneously. As mentioned above, the accelerating electric field is characterized by $E \sim T_{\text {hot }} / e l_{0}$, where $T_{\text {hot }}$ is the temperature of the hot electron and $l_{0}$ is the scale length of the plasma on the rear surface, and is in the order of megavolts per micrometer. A shallow, wavelike surface, such as for the roughened gold targets, is expected to lead to a microlensing phenomenon, consistent with the observed filamentation or spatial modulation of the accelerated protons.

Such microfocusing effects have been calculated for the case of a single concave depression of the surface [7]. In the case of a destroyed surface, the cracks and defects on the plastic and glass create many sharp excursions, very different from the rather smooth undulating surface of the gold targets. The ion plasma created by the field is therefore extended over a much larger scale length normal to the (average) surface. We expect this to partially compensate the charge separation sheath created by the hot electrons, and therefore strongly suppress the ion acceleration.

As mentioned above, it was found in recent experiments [6] that the proton yield of plastic targets was higher than the yield of hydrocarbon contaminants on gold targets, whereas the beam quality from metal targets is much better than from glass or plastic targets [24]. We attempt to increase the yield of laser-accelerated protons, while maintaining the superior beam quality, by adding hydrogencontaining layers of acrylic $(\mathrm{CH})$ to the rear surface of gold targets. We varied the thickness of a $\mathrm{CH}$ layer between 5 and $100 \mathrm{~nm}$. The results showed an increase of the proton yield according to the $\mathrm{CH}$ thickness while maintaining the beam quality. However, at a layer thickness of $100 \mathrm{~nm}$ we observed the onset of filamentlike structures in the spatial distribution of the accelerated protons.

We modeled the response of the RCF package with the SRIM [19] stopping power tables, assuming the beam to be protons, and obtained response functions for the layers of RCF similar to the ones presented in [6]. Taking into account the respective laser pulse energy in these 
experiments, we obtained a conversion efficiency of laser energy into protons of $1 \%$ for the $5 \mathrm{~nm}$ coating and $2.5 \%$ for the $100 \mathrm{~nm}$ coated target.

Finally, the increase of the proton yield was limited by the laser energy available in our experiments. At a given maximum laser energy of $30 \mathrm{~J}$ and a conversion efficiency into hot electrons less than $35 \%$, a layer thickness of several nanometers is sufficient to provide enough protons to be accelerated. This is quite different for petawatt laser systems operating at higher pulse length and accordingly higher total laser energy. In that case an increase in the overall proton yield by at least an order of magnitude can be expected.

A well-known technique to determine the total yield of fast protons is to use nuclear reactions in a catcher material. For our proton beam, we used the ${ }^{48} \mathrm{Ti}(p, n)^{48} \mathrm{Va}$ reaction that provided a sharp threshold at proton energies of $\sim 5 \mathrm{MeV}$. The total yield of ${ }^{48} \mathrm{Va}$ activations produced in a typical shot was $10^{7}$. From that we deduce a total flux of $10^{11}$ laser-accelerated protons, assuming a Maxwellian energy distribution with a temperature of $2 \mathrm{MeV}$. This represents a total conversion efficiency of less than $1 \%$ of the laser energy to accelerated protons.

It is interesting to note that there is an empirical indication of an efficiency scaling with respect to the laser energy. Experiments at the petawatt laser system $\left(500 \mathrm{~J}, 10^{20} \mathrm{~W} / \mathrm{cm}^{2}\right)$ obtained conversion efficiencies of more than $10 \%$ [6], as mentioned in the Introduction. At the Vulcan laser at the Rutherford Appleton Laboratory $\left(100 \mathrm{~J}, 5 \times 10^{19} \mathrm{~W} / \mathrm{cm}^{2}\right)$; conversion efficiencies of a few percent have been determined, we found an efficiency less than $1 \%$ in our experiments at the LULI $100 \mathrm{TW}$ laser $\left(30 \mathrm{~J}, 3 \times 10^{19} \mathrm{~W} / \mathrm{cm}^{2}\right)$, and various laser systems at lower energy obtained conversion efficiencies well below this value.

\section{Proton beam shaping}

An important question to be addressed for any future application of laser-accelerated protons and ions is the possibility of tailoring the proton beam, either collimating or focusing it, by changing the geometry of the target surface. Ballistic focusing of the laser-accelerated protons is expected to be rather difficult because of the inherent divergence associated with the spatial density dependence of the hot electron sheath, which drives the acceleration. Accordingly, we first attempted to defocus the beam in one dimension by using a convex target. Using a $60 \mu \mathrm{m}$ diameter $\mathrm{Au}$ wire as a target basically constituted such a one-dimensional defocusing lens, and we observed a line as shown in Fig. 4. Tilting the wire also changed the orientation of the line, which results from the radial, fan-shaped expansion of the protons normal to the wire.

We then attempted to focus the protons by modifying the curvature (concave) of the target foil. Focusing laser generated protons is essential for many applications such as

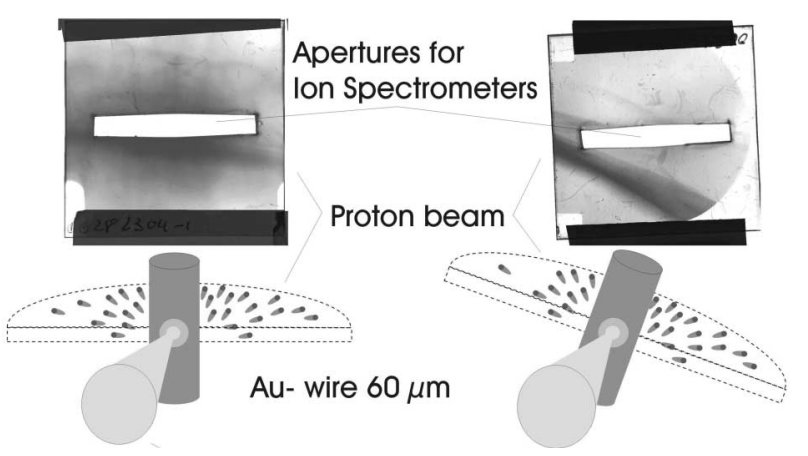

FIG. 4. Experimental setup and RCF images of experiments with $60 \mu \mathrm{m}$ gold wires. The convex rear surface constitutes a decollimating cylinder lens. Accordingly, the proton beam was formed into a line.

ion-induced material damage research, proton driven fast ignition [25], proton radiography [26], and the use as next generation ion sources. Because of the Gaussian-like shape of the hot electron Debye sheath that causes the acceleration, there is an energy dependent angle of divergence that has to be compensated to focus the ions in the energy range of interest. Therefore the effective focal length of a curved target rear surface is longer and is dependent on the proton energy. The results, which will be published elsewhere, show a strong reduction in the divergence of the central core of the proton beam representing ballistic collimating of laser produced proton beams.

\section{E. Heavy ion beam production}

Having established that one can enhance the proton yield while maintaining the beam quality, and at least contemplate successful focusing targets, we next attempted to control the accelerated ion species and, in particular, selectively accelerate either protons or heavy ions. Because of their larger charge-to-mass ratio, which causes the protons to outrun the other ion species during the ambipolar expansion, protons are accelerated faster taking most of the energy from the electrostatic sheath. Therefore, the amount of protons had to be reduced significantly. For targets of solid metals (gold, aluminum), the majority of the protons is due to water vapor and hydrocarbons at the target surface. We reduced these impurities by resistively heating the targets. The targets consisted of thin foils of $50 \mu \mathrm{m} \mathrm{Al}$ coated with $1 \mu \mathrm{m}$ of carbon. To detect the heavy ions with respect to their momentum and charge-state distribution, we substituted the ion spectrometers with two Thompson parabolas at an angle of $0^{\circ}$ and $13^{\circ}$. The ions were recorded in CR-39 plastic track detectors. We compared the yield for heated and nonheated targets, as shown in Fig. 5. As expected, for the nonheated targets (left-hand side) a strong proton signal was observed together with a weak signal of carbon ions. The result changed dramatically for the heated targets, as shown in the right-hand side of Fig. 5. 


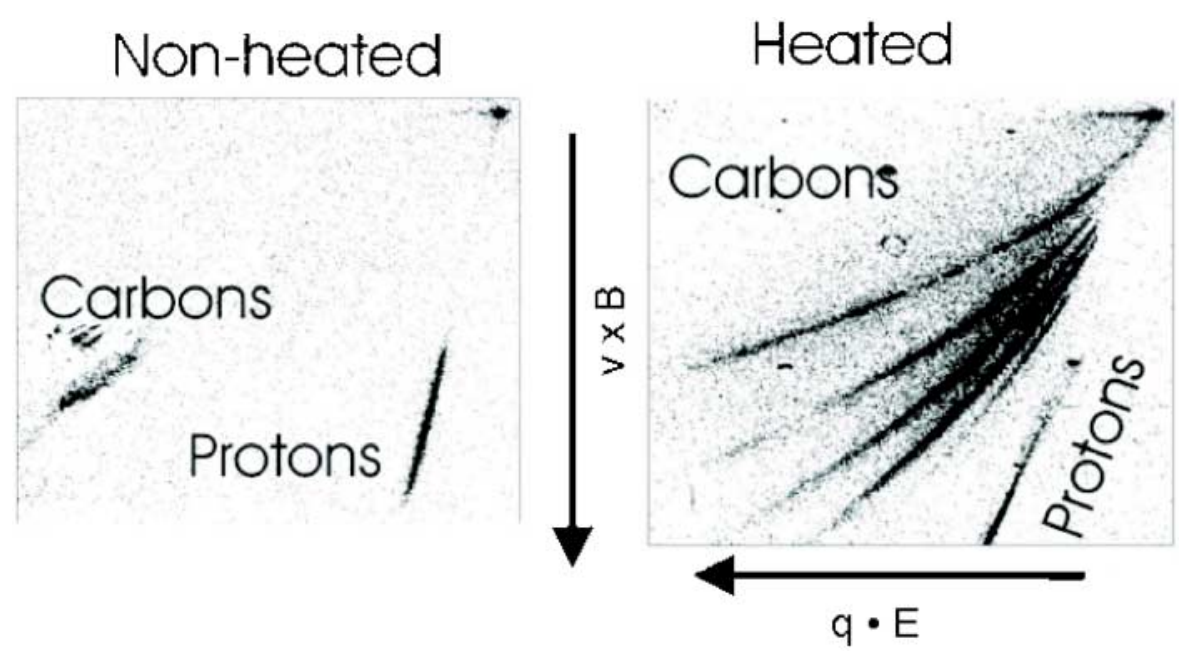

FIG. 5. Heavy ion beam production. In contrast to the strong proton signal (left), removing the hydrocarbons from the target rear surface results in a strong heavy ion (carbon) signal (right).

A sharply reduced proton signal was detected in these experiments together with a much more intense heavy ion signal (carbon and aluminum ions). We observed a higher yield, much higher ion energies and ions at higher charge states. These results are in agreement with experiments using $\mathrm{CO}_{2}$ lasers more than 20 years ago in which removal of hydrogen contaminants by heating increased the ion yield in laser ablation plasmas. In extension to the results obtained in [8], the use of short-pulse chirpedpulse-amplification lasers allows higher focused intensities at shorter pulse length leading to electron temperatures of several $\mathrm{MeV}$, and, consequently, the observed heavy ions are accelerated up to the several $\mathrm{MeV} / \mathrm{u}$ range.

\section{E. Neutron production}

Complementing the laser and ion beam diagnostics we also measured the neutron emission caused by $(\gamma, n)$ and $(p, n)$ reactions from the target. We used a silver activation detector attached to a PMT. On typical shots, the neutrons are generated by $(\gamma, n)$ reactions within the target (caused by the bremsstrahlung photons from the relativistic electrons) and by $(p, n)$ reactions of our proton beam impacting on the RCF screen. We also used a target of deuterized plastic $\left(\mathrm{CD}_{2}\right)$, which was heated to produce a beam of deuterons. Fielding a $\mathrm{CD}_{2}$ catcher foil behind the target we observed the yield of neutrons from $(d, d)$ fusion reactions. We detected a total yield of $2.8 \times 10^{7}$ neutrons in this experiment, which was at least an order of magnitude above the yield on average shots.

\section{F. Proton beam emittance}

For most of the future applications of laser generated ion beams, the beam quality is the most important characteristic. Especially for the use as an ion source or the application as an inertial confinement fusion ignitor beam, the ion beam emittance is crucial with respect to the ac- celerator structure acceptance or the achievable focus spot size. As is apparent from the radiochromic film data, the angular divergence of the proton jet is rather well defined and decreases with increasing proton energy. This suggests that protons or other light ions accelerated by this mechanism may have a usefully small emittance in the sense of an actual ion beam.

To precisely estimate our emittance, we used penumbral imaging of edges at different distances from the target with the magnetic spectrometers to directly measure the core emittance core of the proton beam. This technique is closely related to the conventional slit-emittance measurements made with apertures and screens at conventional accelerators. We determine the normalized emittance of protons from flat gold foils to be $\sim 0.2 \pi \mathrm{mm} \mathrm{mrad}$, and a factor of at least 2 smaller than the resolution limited measurements we performed on the LLNL PETAWATT (see Ref. [24]). Details of the present measurements, and systematics of the proton emittance versus energy, will be reported elsewhere.

The results of this analysis and subsequent modeling, developing a 2D extension of the model in [27], suggest that we observe a rather cold proton beam, which is smoothly diverging and highly laminar. The trace space of the highest energy protons exhibits a tilted ellipse, whose width ultimately is the characteristic of the ion temperature. From these data, we deduce that the proton temperature is less than $\sim 1 \mathrm{keV}$. From simple electron-ion collisional heating during the expansion, one may expect the ion temperature to be even lower, of order $\sim 100 \mathrm{eV}$.

\section{G. Radiography using laser-accelerated proton beams}

The excellent beam quality of the ion beam is ideally matched to the requirements for imaging techniques. One scheme of particular interest is the use of laseraccelerated protons to radiograph macroscopic samples to study their properties. Because of the different interaction 
mechanism, protons can provide complementary information to more common techniques such as $\mathrm{x}$-ray backlighting. The interaction of swift protons with matter is well known and has been a traditional research area of accelerator laboratories over the past decades. Because of the copious amounts of protons accelerated in a very short time, laser-accelerated protons provide a new diagnostic quality in research of transient phenomena. We performed a first set of experiments to demonstrate the feasibility of these laser-accelerated proton beams for radiography applications. Recently, there have been experiments performed to use laser-accelerated protons for object imaging [28]. In these experiments, a thin target (grid) has been placed very close to the proton source. In contrast to these experiments, where the target was exposed to the electron cloud closely behind the target, we have chosen a different geometry. We used a distance of $5 \mathrm{~cm}$ between the proton source and the target in order to minimize any charging of the target and placed the detector (RCF) close to the object to reduce deflection effects. In our experiments, we used a compound target of different materials to be imaged by the protons. It consisted of a $1 \mathrm{~mm}$ thick epoxy ring structure, several copper wires of $250 \mu \mathrm{m}$ diameter, a hollow cylinder with $300 \mu \mathrm{m}$ walls of steel, several $\mathrm{Ti}$ sheets of $100 \mu \mathrm{m}$ thickness, and a glass hemisphere of $900 \mu \mathrm{m}$ diameter and $20 \mu \mathrm{m}$ wall thickness.

The protons were recorded in multiple layers of RCF to detect the image at different proton energies. Figure 6 shows the radiography of the target for final proton energies of 7.5 MeV. The image basically constitutes a negative image of the areal density of the target. The names of the collaborating institutes have been engraved on the epoxy

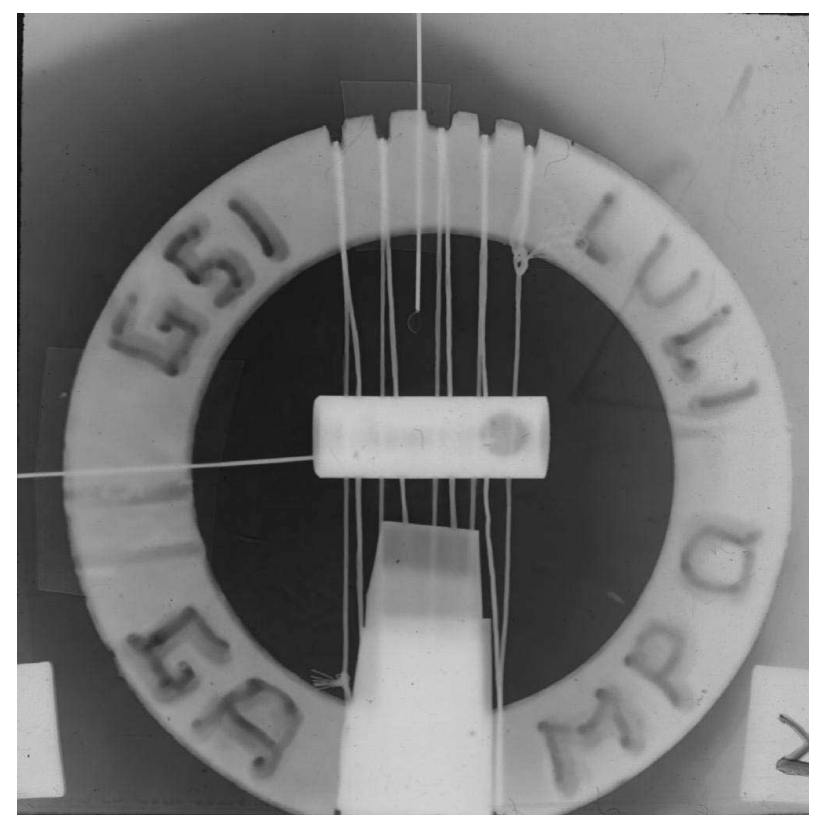

FIG. 6. Radiography of a compound target. Details of the target are given in the text. ring, which results in a reduced thickness and therefore a higher energy deposition of the protons in the respective layer. The areal density variation of the hollow cylinder, including a hole in the wall on the right-hand side, can be seen as well as a thin metal rod placed inside the cylinder. These results show a clear dependence on the areal density rather than on residual charging effects, in contrast to the experimental technique used in [28]. Close examination also shows the small glass hemisphere placed above the cylinder. The time of exposure in this experiment was estimated to be on the order of tens of picoseconds, based on the initial proton beam pulse duration and the energy dependent dispersion of the pulse from the source to the target.

\section{CONCLUSION}

We have presented a detailed investigation of the target conditions on the proton and ion beam production from intense laser solid interactions. The observed strong dependence on the rear surface conditions is in agreement with the target normal sheath acceleration mechanism. The target conductivity appears to have a major influence on the quality of the ion beam, and the quality of the surface finish of the target is very important for maintaining a high gradient sheath and a laminar beam. It has been shown that tailoring the ion beam (yield, shape, composition, homogeneity) by means of target shape and composition is possible, and we present first observations of laser-accelerated ion beam focusing. Finally, the successful generation of a heavy ion beam (carbon, aluminum) further encourages speculation that laser-accelerated ion beams may become a useful tool in a variety of future applications.

\section{ACKNOWLEDGMENTS}

This work was supported by the EU, Contract No. HPRI CT 1999-0052, and in part by Grant No. E1127 from Région Ille-de-France.

[1] A. P. Fews, P. A. Norreys, F. N. Beg, A. R. Beg, A.E. Dangor, C. N. Danson, P. Lee, and S. J. Rose, Phys. Rev. Lett. 73, 1801 (1994).

[2] K. Krushelnick, E. L. Clark, Z. Najmudin, M. Salvati, M. I. K. Santala, M. Tatarakis, A. E. Dangor, V. Malka, D. Neely, and R. Allott, Phys. Rev. Lett. 83, 737 (1999).

[3] A. Maksimchuk, S. Gu, K. Flippo, D. Umstadter, and V. Yu. Bychenkov, Phys. Rev. Lett. 84, 4108 (2000).

[4] M. Zepf, E. L. Clark, K. Krushelnick, F. N. Beg, C. Escoda, A. E. Dangor, M. I. K. Santala, M. Tatarakis, I. F. Watts, and P. A. Norreys, Phys. Plasmas 8, 2323 (2001).

[5] M. Perry and G. Mourou, Science 264, 917 (1994).

[6] R. Snavely et al., Phys. Rev. Lett. 85, 2945 (2000).

[7] S. C. Wilks et al., Phys. Plasmas 8, 542 (2001).

[8] S. J. Gitomer et al., Phys. Fluids 29, 2679 (1986). 
[9] Y. Kishimoto et al., Phys. Fluids 26, 2308 (1983).

[10] H. Ruhl et al., Plasma Phys. Rep. 27, 363 (2000).

[11] A. Pukhov, Phys. Rev. Lett. 86, 3562 (2001).

[12] M. G. Haines, Phys. Rev. Lett. 47, 917 (1981).

[13] M.H. Key, M.D. Cable, T.E. Cowan, K. G. Estabrook, B. A. Hammel, S. P. Hatchett, E. A. Henry, D. E. Hinkel, J.D. Kilkenny, and J.A. Koch, Phys. Plasmas 5, 1966 (1998).

[14] G. Malka and J. L. Miquel, Phys. Rev. Lett. 77, 75 (1996).

[15] F. N. Beg, A. R. Bell, A.E. Dangor, C. N. Danson, A. P. Fews, M. E. Glinsky, B. A. Hammel, P. Lee, P. A. Norreys, and M. Tatarakis, Phys. Plasmas 4, 447 (1997).

[16] M. Tabak, J. Hammer, M. E. Glinsky, W. L. Kruer, S. C. Wilks, J. Woodworth, E. M. Campbell, and M. D. Perry, Phys. Plasmas 1, 1626 (1994).

[17] C. Deutsch, H. Furukawa, K. Mima, M. Murakami, and K. Nishihara, Phys. Rev. Lett. 77, 2483 (1996).

[18] D. Batani, J. R. Davies, A. Bernardinello, F. Pisani, M. Koenig, T. A. Hall, S. Ellwi, P. Norreys, S. Rose, and A. Djaoui, Phys. Rev. E 61, 5725 (2000).
[19] J. F. Ziegler, J. P. Biersack, and U. Littmark, The Stopping and Range of Ions in Solids (Pergamon, New York, 1996).

[20] R. Ramis, R. Schmalz, and J. Meyer-ter-Vehn, Comput. Phys. Commun. 49, 475 (1988).

[21] A. J. MacKinnon, M. Borghesi, S. Hatchett, M. H. Key, P. K. Patel, H. Campbell, A. Shiavi, R. Snavely, S. C. Wilks, and O. Willi, Phys. Rev. Lett. 86, 1769 (2001).

[22] L. B. Lucy, Astron. J. 79, 745 (1974).

[23] F. Pisani, A. Bernardinello, D. Batani, A. Antonicci, E. Martinolli, M. Koenig, L. Gremillet, F. Amiranov, S. Baton, and J. Davies, Phys. Rev. E 62, R5927 (2000).

[24] T.E. Cowan et al. (to be published).

[25] M. Roth et al., Phys. Rev. Lett. 86436 (2001).

[26] A. Blazevic et al. (to be published).

[27] L. M. Wickens and J.E. Allen, Phys. Fluids 24, 1984 (1981).

[28] M. Borghesi, A. Shiavi, D. H. Campbell, M. G. Haines, O. Willi, A. J. MacKinnon, L. A. Gizzi, M. Galimberti, R. J. Clarke, and H. Ruhl, Plasma Phys. Controlled Fusion 43, A267 (2001). 